\title{
QUANTITATIVE ISOLATION OF CHLOROPLASTS FROM HIGHER PLANTS ${ }^{1}$
}

\section{Sam Granick}

Chloroplasts, because of their fundamental role in photosynthesis and their prominence in leaf cells, have been the object of many cytological and microchemical studies. A considerable and controversial literature has accumulated on the constitution of chloroplasts, containing a large number of conjectures and a paucity of facts-a condition arising from the limitations of the methods that have been used.

The writer has found that chloroplasts can be isolated by differential centrifuging in amounts sufficient for quantitative determinations. Once the isolation is made, analytical procedures can be taken almost bodily, from the field of blood chemistry. It is hoped that the quantitative isolation of chloroplasts will open up a new and more fruitful field of investigations, not only on the composition, structure, and metabolism of these bodies, but also on the mechanism of photosynthesis.

FaCtors INYOLVED IN THE ISOLATION OF CHLOROPLASTS. - During preliminary studies of leaf protoplasm it was noted that partial separations could be made of its components by centrifugation. Examination of the centrifugates of a tissue mash prepared by grinding leaves in $0.5 \mathrm{M}$ glucose solution indicated that the cell wall debris and the unbroken cells were thrown down first, followed in order by the larger crystals, and starch grains torn from the chloroplasts, coagulated cytoplasm, smaller crystals and starch grains, chloroplasts, and finally by very tiny granules. This led to an attempt to isolate chloroplasts in as normal a state and with as little contamination as possible.

It is intended in a later paper to discuss in detail the composition and morphological structure of the chloroplasts. It will suffice here to mention briefly a few facts relevant to the problem at hand. Chloroplasts of tomato and tobacco leaves possess a semipermeable membrane and can be maintained for a time in a solution. which is isotonic or hypertonic with that of the cytoplasm of the leaf cell. Of the various substances tested, $0.5 \mathrm{M}$ glucose or sucrose solutions were found to be the most satisfactory in which to disperse the chloroplasts. These solutions do not coagulate the cytoplasmic proteins nor cause coarse granules to appear in the chloroplasts, as do salt solutions in general. The chloroplasts obtained in this manner remain normal for several hours as judged from observations on the presence of functional semi-permeable membranes, although very fine

${ }^{1}$ Received for publication May 24, 1938.

This is the first of a series of papers on the structure and metabolism of chloroplasts.

Papers from the Department of Botany of the University of Michigan, No. 668.

The author desires to express his appreciation to Professor F. G. Gustafson for his interest and advice in this investigation. granules become visible in them after a short time. They have been found to produce oxygen for several minutes in the presence of light, as determined on individual chloroplasts by Engelmann's motile-bacteria method. The nuclei, at the $\cdot \mathrm{pH}$ of the tissue suspensions ( $\mathrm{pH}$ 6.0-6.5), disintegrate completely and so do not come down with the chloroplasts on centrifuging.

In order to decide whether careful microscopic observation of the centrifugate might be used as a criterion of the purity of the chloroplasts which had been separated out, analyses were first made on the nitrogen content of these bodies. It will become clear further on that because of the method of analysis used, if cytoplasmic proteins were carried down with the chloroplasts, a value for the percentage of $N$ of the chloroplasts in a cell as compared with that of the total $N$ per cell might well exceed 100 per cent. From the nitrogen analyses it was found that the lowest and most consistent $N$ values were obtained for the cleanest chloroplast centrifugates as revealed by microscopic examination under an oil-immersion lens. It appears therefore that direct microscopic examination is a reliable method for checking the purity of the isolated chloroplast fraction. Furthermore, certain experiments now in progress on the enzyme content of chloroplasts lead the writer to the belief that there is relatively little contamination of the isolated chloroplasts with other proteinaceous materials of the protoplasm.

No definite statement can be made concerning the rate of centrifuging, since this was found to depend on the age and kind of leaf that was used. However, it may be said that as a general rule centrifugation at 400 to 800 times gravity was found satisfactory.

The method is not applicable to the extremely young leaves in which cell division is still taking place, because it is difficult to isolate the small ehloroplasts from the protoplasm. Also, determinations cannot be made on the oldest leaves which are yellow and have degenerating chloroplasts, since not only does the protoplasm appear to coagulate very readily, but the chloroplasts have too little pigment to permit of their quantitative estimation by use of the colorimeter. These factors likewise make the analyses of the earliest and latest stages of growth somewhat less accurate than those made on leaves that have just attained their maximum size. A further limitation of the method is the fact that it is necessary to choose leaves whose cell walls are readily broken by maceration in sand so that the chloroplasts can float out into the sugar solution. Of a number of leaves tested, those of tomato and tobacco were found especially suitable.

Factors involved in the quantitative estimaTION OF THE CHLOROPLASTS.-In order to determine the percentage of the chloroplasts isolated, one may 
count the number of chloroplasts in the centrifugate and compare this count with the number of chloroplasts in the gram of leaf. Such a method is obviously impractical. An indirect method is used for this determination which takes advantage of the fact that the chloroplast pigments are present solely in the chloroplasts. It is assumed that the quantity of chloroplasts is directly-proportional to the concentration of chloroplast pigments contained in them. This assumption is valid for all practical purposes since we are dealing with a large number of chloroplasts. In order to illustrate this principle, let us suppose that a fraction, obtained from one gram of leaf, is centrifuged down and on microscopic examination is seen to contain only chloroplasts. An acetone extract is then made of these chloroplasts. At the same time, one gram of leaf material is also extracted with acetone. On comparing the extracts, it is found, let us say, that the extract of chlorophyll pigments of the isolated chloroplasts contains onetenth of the chlorophyll pigments in one gram of leaf. Then it is justifiable to assume that ten per cent of the chloroplasts in one gram of leaf are present in the centrifuged fraction.

The same principle is utilized in another way. Suppose a suspension of chloroplasts is centrifuged for a short time. Then a certain amount of the chloroplasts will be thrown to the bottom of the tube. If the concentration of chloroplast pigments in the suspension is determined before and after centrifuging, then the concentration of chloroplast pigments in the centrifugate may be calculated.

In order to determine the concentration of plastid pigments, extractions of the suspensions are made with 75 per cent acetone, which has been found to be better than a number of other solvents for this purpose. The acetone dissolves the pigments of the chloroplasts and at the same time produces a heavy flocculent precipitate of the proteins. On centrifuging, a clear green supernatant solution results which can be used for colorimetric determinations. A clear solution of the pigments is essential, since the accuracy of the chloroplast values depends on the accurate determination of the concentration of the pigments in the aliquots of the chloroplast suspensions before and after centrifuging.

Although preliminary experiments showed that the acetone extracts of the pigments of most leaves fade slowly, the fading was found to be directly proportional to the concentration of the pigments. Since the method is not concerned with the absolute but with the relative concentrations of the pigments, this introduces no error. However, experience with a large number of analyses has indicated that occasionally the fading of acetone extracts of the pigments is not proportional to the concentration. In order to avoid errors due to fading, it has been found best to make extractions with ice cold acetone and to determine the pigment concentrations immediately after the preparation of the acetone extracts. Extracts of the pigments can also be made with a $3: 1$ alcohol-ether mixture. These extracts fade less rapidly than do the acetone extracts but sometimes do not give as clear solutions.

Sample analusis. - An example from a typical analysis will best illustrate the method of isolating the chloroplasts and the method of calculating the fraction of the total chloroplasts that has been isolated. Of the various modifications of procedure tested, experience suggests the following ones as the most satisfactory:

An average sample of three grams of fresh leaf tissue, excluding midribs and main veins, is weighed out rapidly on a torsion balance. The tissue is then kept between moist toweling paper until ready for analysis. During the interim, the cells absorb water, becoming turgid, and in this condition are more readily torn apart. Analyses are generally begun within an hour after the leaves have been cut from the plants.

The leaf tissue is removed from the moist toweling paper, washed once with distilled water, superficially dried, and a portion of it placed in a deep porcelain mortar of about $150 \mathrm{cc}$. capacity. One gram of quartz sand and $25 \mathrm{cc}$. of $0.5 \mathrm{M}$ glucose solution at a temperature of about $5^{\circ} \mathrm{C}$. are added to the mortar. The tissue is then rubbed gently with the pestle against the sand in order to tear the turgid cells apart and release the chloroplasts into the cold hypertonic glucose solution. When the solution has become dark green, the flaccid tissue is squeezed against the side of the mortar, and another portion of tissue is added and the cells torn apart in a similar manner. The chloroplast suspension is decanted into a $50 \mathrm{cc}$. round-bottom centrifuge tube. The remainder of the tissue is then ground in an additional $20 \mathrm{cc}$. of the cold glucose solution, and this chloroplast suspension is added to the first in the centrifuge tube. Thus, a total of approximately $45 \mathrm{cc}$. of a green suspension is obtained. (The leaf residues remain in the mortar and are treated as mentioned below.)

As mentioned above, the time and rate of centrifugation that are used in the isolation of the chloroplasts depend on the leaf material under investigation. These factors must be controlled by careful examination of the centrifugates under an oil-immersion lens. The time intervals for centrifuging that follow are taken from a typical analysis.

The suspension is centrifuged for some seven minutes at about 400 times gravity in order to throw down any heavy cellular debris, crystals, and large starch granules. The supernatant suspension is then decanted into another centrifuge tube and diluted up to a $45 \mathrm{cc}$. mark with glucose solution. This suspension is designated as suspension A. Five cc. of suspension $\mathrm{A}$ are pipetted into a tapering-bottom centrifuge tube of 20 to 25 cc. capacity for the determination of the concentration of chloroplast pigments.

The debris remaining in the first centrifuge tube is transferred to the mortar and, together with the leaf residues, is ground up very thoroughly in glucose solution and made up to $50 \mathrm{cc}$. in a volumetric flask. 
This tissue mash is designated as suspension $\mathrm{D}$. Five cc. of this suspension are pipetted into a centrifuge tube for the determination of its concentration of chloroplast pigments.

The remaining $40 \mathrm{cc}$. of suspension $\mathrm{A}$ are centrifuged for five to eight minutes. The resulting supernatant suspension is then decanted into another centrifuge tube and is designated as suspension $\mathrm{B}$. The chloroplasts which have been thrown down are designated as centrifugate $B$ and serve for the direct analysis of nitrogen, phosphorus, or enzyme, etc. Five ce. of suspension B are pipetted into a centrifuge tube for the determination of the concentration of chloroplast pigments in suspension B.

The 35 cc. of suspension $B$ remaining are further centrifuged for five to ten minutes, the supernatant suspension decanted into a test tube and designated as suspension $\mathrm{C}$. The chloroplasts that have been centrifuged down are designated as centrifugate $\mathrm{C}$ and serve for direct analysis. Five cc. of suspension $\mathrm{C}$ are pipetted into a centrifuge tube for the determination of the concentration of chloroplast pigments.

To each of the 5 cc. samples of suspensions A, B, C, and $\mathrm{D}$, which have been transferred to the taperingbottom centrifuge tubes of 20 ta $25 \mathrm{cc}$. capacity, are added 10 to $15 \mathrm{cc}$. of ice cold aceton. By properly tapping the tubes to set up a swirling motion, the solutions will be found to mix thoroughly. They are placed in a beaker containing some ice and water in a dark place for some ten to fifteen minutes. Then the tubes are stirred again and centrifuged rapidly for five minutes. Crystal-clear green solutions result. These are decanted into 25 cc. volumetric flasks, marked respectively $\mathrm{A}, \mathrm{B}, \mathrm{C}$, and $\mathrm{D}$, which are placed in ice water. The residues in the centrifuge tubes are extracted once more with $5 \mathrm{cc}$. of 75 per cent acetone and the extracts added to the main volume of extracts in the flasks. The green solutions are compared in a Duboseq biological colorimeter. Since much depends upon the accuracy of the readings of the colorimeter, the instrument should be carefully adjusted and duplicate readings should not vary by more than $0.2 \mathrm{~mm}$. on the scale. Beer's law is obeyed within the range of concentrations that are used.

Comparisons are made between solutions $\mathrm{A}$ and $\mathrm{B}$, $B$ and $C$, and $A$ and $D$. The scale readings of the cups of the colorimeter are given for a typical analysis: $\mathrm{A} / \mathrm{B}=21.1 / 30.0=0.702 ; \mathrm{B} / \mathrm{C}=20.4 / 30.0=$ $0.679 ; \mathrm{A} / \mathrm{D}=20.0 / 21.1:=0.942$.

Cal,culation of the percentage of the chloroPLASTS PRESENT in CENTRIFUGates B and C. - In order to calculate the percentage of the chloroplasts present in centrifugates $\mathrm{B}$ and $\mathrm{C}$, it is necessary to know the percentage of the pigments present in suspension A. This may be determined in the following manner:

All the blade material taken for analysis $(3.0 \mathrm{~g}$.) is present in 45 cc. of suspension $\mathrm{A}+50 \mathrm{cc}$. of suspension D. Consider that the amount of pigments in the sample is 100 per cent. Let the concentration of pigments in $1 \mathrm{cc}$. of suspension $\mathrm{A}=1.0$ unit.
Now $\mathrm{A} / \mathrm{D}=0.942$-i.e., 1 cc. of suspension $\mathrm{D}$ is only 0.942 times as concentrated as $1 \mathrm{cc}$. of suspension A. Then $\mathrm{A}=1.0 \times 45 \mathrm{cc} .=45.0 ; \mathrm{D}=0.942 \times 50 \mathrm{cc} .=$ 47.2 and the sum i.e., $45.0+47.2=92.2$ units $\approx$ 100 per cent of the pigments. The percentage of the pigments or of the chloroplasts which are present in 45 cc. of suspension $\mathrm{A}=45 \times 100 / 92.2=48.8$ per cent. Before suspension $A$ is centrifuged, 5 cc. are removed, leaving only $40 \mathrm{cc}$. of A. The percentage of chloroplasts present in $40 \mathrm{cc}$. of $\mathrm{A}$ is $40 / 45 \times 48.8=43.4$ per cent. After centrifuging, the percentage of chloroplasts present in $40 \mathrm{cc}$. of suspension $\mathrm{B}=\mathrm{A} / \mathrm{B} \times 43.4$ or $0.702 \times 43.4=$ 30.5 per cent. The percentage of chloroplasts in centrifugate $B$ is equal to the difference in the percentage of pigments before and after centrifuging the 40 cc. of chloroplast suspension - i.e., 43.4 per cent -30.5 per cent $=12.9$ per cent. Centrifugate $B$ therefore contains 12.9 per cent of the total number of chloroplasts present in three grams of leaf.

The percentage of chloroplasts in centrifugate $\mathrm{C}$ is calculated in a similar manner. In 40 cc. of suspension $\mathrm{B}, 30.5$ per cent of the chloroplasts are present. In $35 \mathrm{cc}$. of suspension B, 26.7 per cent of the chloroplasts are present. After centrifuging, suspension C contains $0.679 \times 26.7$ per cent $=18.1$ per cent of the chloroplasts. The percentage of chloroplasts in centrifugate $\mathrm{C}$ is then $26.7-18.1=8.6$ per cent. Centrifugate $C$ therefore contains 8.6 per cent of the total number of chloroplasts present in three grams of leaf.

An outline of the method for the quantitative isolation of chloroplasts is given in the accompanying diagram.

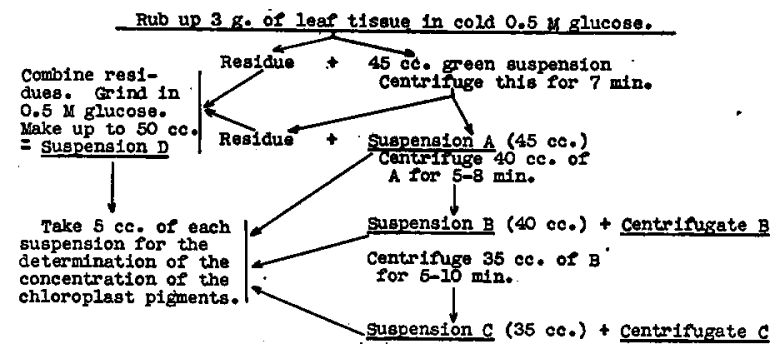

An example, taken from some Kjeldahl nitrogen determinations will illustrate the method of calculating the percentage of nitrogen in the chloroplasts (table 1). The table is self-explanatory.

If nitrogenous substances, which are not a part of the chloroplasts, are carried down with them, it will generally be found that centrifugate $B$ will give a higher value for the percentage of nitrogen in the chloroplasts as compared to centrifugate $\mathrm{C}$. The two centrifugates serve as a check against each other. It will be observed from the table that the values obtained from both. centrifugates, as indicated in the last column, check each other within the limits of the experimental error of the procedure.

A second procedure, which is also quite satisfactory, is the following: About ten grams of leaf blade 
material are ground up to release the chloroplasts, as described above. A first short centrifuging removes the debris. A second more rapid and prolonged centrifuging carries down most of the chloroplasts.

These chloroplasts are then resuspended in glucose solution and centrifuged for a short time to remove clumps of chloroplasts. A second, more prolonged centrifuging, carries down most of the chloroplasts in a relatively pure condition. the cytoplasmic proteins coagulate. The chloroplasts are separated from other cell materials by a system of differential centrifuging.

A method is presented for relating the quantity of chloroplasts isolated to the total quantity of chloroplasts in the leaf sample. The method is based on the assumption that the number of chloroplasts is directly proportional to the quantity of chloroplast pigments contained in them. By determining the

TABLE 1. Method of calculating percentage of nitrogen in chloroplasts.

\begin{tabular}{|c|c|c|c|c|}
\hline Material & $\begin{array}{l}\text { \% of the } \\
\text { chloroplasts } \\
\text { used for } \\
\text { analyses }\end{array}$ & $\begin{array}{l}\mathrm{N} \text { in the } \\
\text { chloroplasts }\end{array}$ & $\begin{array}{l}\mathrm{N} \text { in } \\
\text { all }(100 \%) \\
\text { of the } \\
\text { chloroplasts }\end{array}$ & $\begin{array}{l}\% \text { of the } \\
\text { total- } \mathrm{N} \text { in the } \\
\text { leaf blade material } \\
\text { which is present } \\
\text { in the chloroplasts }\end{array}$ \\
\hline Centrifugate & & . & & \\
\hline B $\ldots \ldots \ldots$ & $12.9 \%$ & $0.917 \mathrm{mg}$. & $7.12 \mathrm{mg}$. & $43.8 \%$ \\
\hline $\mathrm{C} \ldots \ldots \ldots$ & 8.6 & 0.578 & 6.72 & 41.5 \\
\hline
\end{tabular}

The cleaned chloroplasts are then suspended in distilled water and analyses are made on them. An aliquot of the suspension is taken for the determination of the concentration of chloroplasts in the suspension. The aliquot is treated with acetone in a centrifuge tube, and the contents of the extracted chloroplast pigments are compared in a colorimeter with the pigments extracted from one gram of fresh leaf material, as described above. The number of chloroplasts in the washed suspension can thus be related to the number of chloroplasts present in one gram of fresh leaf-blade material.

\section{SUMMARY}

A procedure is described for isolating chloroplasts from tomato and tobacco leaves. The cells of the leaves are torn apart by grinding the cells under hypertonic sugar solutions. The suspended chloroplasts in these solutions do not disintegrate, nor do amount of chloroplast pigment in a fraction of isolated chloroplasts and relating this value to the amount of chloroplast pigment in the entire tissue sample, one may obtain the percentage of pigment present in the fraction of isolated chloroplasts. For practical purposes this will also be the percentage of chloroplasts which is isolated from the tissue sample.

A certain quantity of chloroplasts are isolated in a relatively pure state from three grams of leaf material, and the pigments of these chloroplasts are extracted with 75 per cent acetone. The quantity of pigments extracted from them is then compared colorimetrically by means of a Duboscq colorimeter with the quantity of pigments extracted from three grams of leaf material. From this value one may obtain the percentage of the chloroplasts which have been isolated from three grams of leaf material.

\footnotetext{
Department of Botany,

UNIVERSITY OF MichigaN, ANN ARBor, Mich.
}

\section{CHLOROPLAST NITROGEN OF SOME HIGHER PLANTS ${ }^{1}$}

\section{Sam Granick}

IN THE first paper of this series (Granick, 1938) a method was described by which chloroplasts can be isolated quantitatively. In the present paper, the method is applied to the analyses of the nitrogenous composition of these bodies. Data on the nitrogenous constituents, especially the proteins, have long been desired in order to answer several important questions concerning the physiology of these bodiesnamely: To what extent are the proteins of a cell

1 Received for publication May 24, 1938.

This is the second of a series of papers on the structure and metabolism of chloroplasts.

Papers from the Department of Botany of the University of Michigan, No. 669 .

The author desires to take this opportunity to express his gratitude to Professor F. G. Gustafson for his advice and interest in these investigations. present in the chloroplasts? Are the chloroplasts significant synthesizers of proteins? What is the relation between the protein of the chloroplasts and chlorophyll?

The investigations as far back as Sachs (1862) and the more recent ones of Molisch (1916), Lakon (1916), Meyer (1918), Ullrich (1924), and others indicated that the major portion of the cell proteins of the leaves was present in the chloroplasts. The changes observed in the size of these bodies on supplying starved leaves with a carbohydrate and nitrogen source, or accompanying the aging of leaves, led these workers to the further conclusion that the major portion of the cell proteins of the leaves is synthesized by the chloroplasts. Schumacher (1929) has recently criticized the above mentioned investigations 